\title{
A Systematic Design Approach in Building Digitalization Services Supporting Infrastructure
}

\author{
Pavel Petrov ${ }^{1}$, Mihail Radev ${ }^{1}$, Georgi Dimitrov ${ }^{2}$, \\ Adrian Pasat ${ }^{3}$, Alexander Buevich ${ }^{4}$ \\ ${ }^{1}$ University of Economics - Varna, Varna, Bulgaria \\ ${ }^{2}$ University of Library Studies and Information Technologies, Sofia, Bulgaria \\ ${ }^{3}$ Beia Consult International, Bucharest, Romania \\ ${ }^{4}$ Ural Federal University, Institute of Industrial Ecology UB RAS, Ekaterinburg, Russia
}

\begin{abstract}
The creation of an efficient digital infrastructure is a prerequisite for the successful operation of any digital service with a high degree of complexity. In the best case, the organization of information processes should be based on the best practices in the construction of digital infrastructure, which allows the future development. With increasing the level of integration of digitalization with daily company activities, it is necessary to pay more attention to the problems of ensuring the continuity of digitalization processes. There are a lot of methods that have been developed to select the best of multiple options, each of which evaluated on the base of multiple criteria. In this research the usage of one of them is proposed as most suitable for taking decisions on building digital infrastructure for digitalization services.
\end{abstract}

Keywords - digital infrastructure, digitalization services, decision making, digitalization management, digital security

DOI: 10.18421/TEM101-04

https://doi.org/10.18421/TEM101-04

Corresponding author: Pavel Petrov,

University of Economics - Varna, Varna, Bulgaria.

Email: petrov@ue-varna.bg

Received: 22 October 2020.

Revised: 03 December 2020.

Accepted: 10 December 2020.

Published: 27 February 2021.

(cc) BY-NC-ND (C) 2020 Pavel Petrov et al; published by UIKTEN. This work is licensed under the Creative Commons Attribution-NonCommercial-NoDerivs 4.0 License.

The article is published with Open Access at www.temjournal.com

\section{Introduction}

Modern digital services are provided by hardware and software, offered by a variety of providers where the ability to work together between components is essential to the operation of the system as a whole. We could say that the equipment with software, hardware, and network equipment from different providers is a great challenge in achieving best performance.

According to the good practices about the structure of a system providing digitalization services, the following main system modules could be distinguished: customer service, technical support, data transmission equipment, data storage and archiving, digital security. Each one of them should follow the principle of ensuring the continuity of digitalization processes.

Prior to the development and implementation of each of the system modules, a set of organizational and technical measures must be performed, mainly consisting of:

- A comprehensive and integrated analysis of the functioning of the components of the digital infrastructure and their role in the system as a whole;

- Formal definition of the structure of information services as a basis for the functioning of the system and development of measures for their optimization;

- Planning the development of the technical and digital infrastructure and development of methodologies for its operation;

- Estimating the feasibility of creation of new or renovation of the existing digitalization infrastructure;

- Designing of all processes in accordance with generally accepted standards;

- Feasibility study for proposing solutions; 
- Contracting of the service level agreements (SLAs) with suppliers and providers;

- Applying information technology services management (ITSM) approach;

- Applying a set of measures for commissioning of separate modules for digital services.

It should be noted that the application of information technology services management is a modern approach applied in large corporations. But it also could be successfully applied on a smaller scale. The main idea is that employees and consumers do not focus on the tools used to perform a particular activity, but rather on the benefit and the result. Services can be easily defined and described, and subsequently their performance can be evaluated and improved accordingly.

\section{Organizational Problems in the Processes of Digitalization}

When purchasing a new product or service, it is important to think about the maintenance of digital services in the long term. It is necessary to be provided the following set of activities:

- Warranty and out-of-warranty service of digital services;

- Preventive measures to maintain the operability of digital services in accordance with service level agreements (SLAs) with providers;

- Constant monitoring of the operation of the digitalization infrastructure and provided services;

- User training;

- Guaranteed response time in case of problems and functional replacement of components during repair;

- Outsourcing of digital subsystems.

With increasing the level of integration of digitalization with daily company activities, it is necessary to pay more attention to the problems of ensuring the continuity of digitalization processes. This is because with the development of digitalization, companies are becoming more and more dependent on them and therefore the main task of the digitalization infrastructure is to ensure the continuity of processes. In this regard, various indicators could be used, such as reliability, performance, security of individual components of equipment and digital services in general. One of the goals should always be to increase the reliability of the digitalization infrastructure. This can be successfully achieved by duplicating critical components, so that in case of failure in a short period of time one is able to switch to the use of spare equipment. It should be noted that no matter how much a digital service is designed to be fault tolerant, increasing reliability only reduces the likelihood of failures and such failures cannot be fully eliminated.

The subsystem for ensuring the continuity of digitalization processes is designed to reduce the adverse effects of natural disasters, various accidents, security failures and other emergencies, and is responsible for restoring digitalization in such situations. These events are exceptional for both users and technical support providers, and in this regard the digital continuity subsystem greatly facilitates the recovery process after such incidents.

Problems in the digitalization infrastructure could be considered extraordinary if they are related to the refusal of digitally critical resources or a breach of the service level agreement. In these cases, the subsystem to ensure the continuity of digitization processes must provide for remedial action through a pre-prepared set of measures:

- Periodic and systematic archiving of data. Backing up data is a traditional method of increasing the reliability of data processing systems [1]. The backup procedures must be clearly defined in the digital security subsystem. It is necessary to provide for the storage of backups of all-important data in at least two remote locations. The trends of increasing volumes and frequency of use of digital information require the creation of a separate system for storage and archiving of data. The transition from local hard drives, which are rarely well administered from the backup point of view, to a separate storage subsystem, makes it possible to provide a single information space on multiple servers and applications. In this way, the data storage and archiving subsystem is a network resource that is efficiently intended for sharing by various software and to organize access from all servers to the shared disk space. The infrastructure needed for the implementation is a high-speed network and storage devices to which the servers connect through special applications [2], [3]. The main advantage of the common data storage system is that it centralizes the management and reduces the time for data backup and recovery.

- Maintenance of spare equipment to reduce recovery time. The backup equipment needed to restore the digitalization services in emergency situations shall be used if it is not possible to restore services within the prescribed time, in accordance with their priorities.

- Periodic training for implementation of activities for restoration of the digitalization infrastructure. Testing the recovery plan ensures that employees can act effectively in emergencies. Testing of the 
recovery plan must be carried out at regular intervals or when the recovery plan is changed.

- Continuous training of employees for action in emergency situations. Periodic training of employees is necessary in order to systematize the recovery process in employees and consists in acquainting users with the documents governing the use of digital services and giving basic guidelines for consumer actions in emergencies, as well as the place of conducting exercises to implement a recovery plan.

- Recovery plan for the infrastructure should be made to ensure continuity of digitization services. The recovery plan shall include emergency recovery activities. Each digitization resource is given priority, which determines the requirement to restore the service in each period. The following information should be included: conditions for activating the plan; actions to restore the normal functioning of the components of digitalization; the terms for restoration of the digitalization services; the persons responsible for restoring the functioning of the individual components responsible for digitization.

Recovery time depends on the priority of the components. Usually a high priority component should be restored within a few hours, with a medium priority - up to 1-2 days, with a low priority - up to 1 week. If it is not possible to complete a component recovery within the required time, the recovery generally can take place in two stages:

- Temporary recovery, sufficient to support a large part of the digitization processes without compromising the security of digitization services. The duration of this stage depends on the reasons for the emergency.

- Full restoration of the functioning of the digitalization services with transition to normal operation of all components.

In order to implement the recovery plan, it is necessary to periodically carry out training activities for employees to work in emergency situations, test the recovery plan, as well as its periodic updating to ensure its compliance with the current state of digitalization infrastructure.

The following steps should be followed when the recovery plan should be triggered and implemented:

- a signal of a problem from users or from the digital infrastructure monitoring system is received;

- expert decides on existence of an emergency situation exist and there is need to implement the recovery plan;

- notifying the persons responsible for the implementation of the recovery plan;
- notifying higher management bodies of an emergency.

The digital security subsystem should cover all components of the digitalization infrastructure and should ensure a high level of confidentiality, integrity, and accessibility of information. It should ensure safe operation of the system without interfering with the work of users with information resources.

There are international standards for digital security currently in the development stage, such as ISO/IEC CD 27002.2 "Information security, cybersecurity and privacy protection - Information security controls" and all previous revisions back to ISO/IEC 17799:2000 "Information technology Code of practice for information security management". The standards recommend what issues should be considered when designing a digital security system, covering various aspects of information systems security.

The choice of measures and means to ensure digital security is made based on an analysis of the risks levels [4] to which the components of the digitalization infrastructure are exposed. The main goal is to preserve the confidentiality, integrity, and accessibility of information resources. Loss or violation of some of the above qualities of information should be considered as a violation of digital security. By its nature, the digital security system is an integrated set of administrative and organizational actions and specialized software and hardware with the goal to ensure protection of the components of the digitalization infrastructure from various threats and risks.

For the successful functioning of the digital safety system, the administrative part of the system [5] occupies an important place, which must ensure control over the functioning of the system by creating a digital safety policy that defines the basic principles and scope of action. Policies are also needed for the use of information services, which should define specific requirements for the individual components of the digitalization infrastructure, as well as to develop procedures for ensuring security policies [6], as a set of measures and countermeasures to implement the requirements of security policies and ensuring control over their implementation.

\section{Technological Problems in Digitalization}

Many companies go through cyclical phases of constantly using digitalization in their activities by using the technique of Business Process Modelling [7]. During the first phase, the needs for new digitalization services are determined. During the second phase, hardware (computing, communication, etc. equipment) and software (software - system, 
application, management information systems, etc.) are purchased. During the third phase, a process of maintaining and updating the used hardware and software takes place. Subsequently, the phases are repeated.

The main "driving force" during the individual phases is the financing, as the companies in most cases do not realize revenues that would enable them to support themselves [8]. Despite the indisputable advantages, the process of digitalization has some negative sides - the company's activity increasingly depends on modern technologies and in the overall organization of the work appear the so-called "bottlenecks" - insufficient capacity to maintain a normal pace of work and maintenance of constantly increased complex equipment. This has a significant impact on the normal functioning, in case some delays occur, and synchronicity is lost in the joint work of the individual structural units of the company. As a result, there could be a situation of dissatisfaction with the state of digitalization level, despite the availability of modern hardware and software. The situation is further complicated by the existence of ambiguities on a number of issues - for example, it is not clear how the existing digitalization will develop and evolve, whether it makes sense to invest in new emerging technologies and so on. As a result of this uncertainty, the budget for the supply of new digitalization services cannot be optimized.

To avoid these adverse phenomena, it is necessary for a strategy for the development of digitalization to be developed, which aims to minimize the risks of using and developing digitalization services in the long run [9]. Risks can be very diverse in nature and some of them could be defined as follows: investment risk or potential costs, unauthorized access (security), lack of integration, inconsistent information, interruption of access to information services [10], inefficient infrastructure etc.

On the one hand, these may be reasons related to operational activities - various types of threats to digital security - intentional damage to equipment, hacker attacks, viruses, data theft, etc. On the other hand, these risks may be from tactical nature - in the analysis, design, documentation, purchase and implementation of hardware and software. It is possible not to choose an optimal solution for digitalization, not to fully use the potential of the purchased equipment, to have discrepancies between the built digital infrastructure and the directions of development, to have organizational and technical mistakes while installing various information systems, not be able to maintain and develop the digital system at an acceptable level, no emergency measures are planned, the infrastructure cannot withstand normal or peak loads, suboptimal technical support procedures are applied, etc.
In order to reduce the risk of such threats, which hinder normal activity, and hence reduce work efficiency, it is necessary to create a strategy that defines long-term goals and the "direction of movement" in the field of digitalization. The strategy should enhance the usefulness of the company and its implementation should ensure the successful existence and development of the company in today's conditions of total computerization and digitalization.

To develop a strategy, the management of the company must know the world experience very well and consider the importance of good practices. In this regard, the strategy can range from providing basic support for the existing systems to providing the most up-to-date infrastructure and digitalization services, if this will contribute to improving customer service. The strategy might be a plan to renew computers, change the operating system or develop a website, but above all it should indicate how, based on current technologies, customer service can be improved in order to increase their satisfaction and possibly to attract new users. In some cases, a key task in the strategy may be to reduce the operating costs of digital infrastructure if the company's budget is expected to shrink. Such a scenario is quite possible to meet in the coming years in view of the adverse global cataclysms such as pandemics and other natural disasters that cannot be predicted.

In order for the developed strategy to be effective, it should take into account the needs for services for the next few years, which allows to increase the efficiency of employees, and hence the company as a whole, in terms of the digital services offered. In this sense, it is necessary to apply a business approach. In business, strategy means a management plan aimed at strengthening the position of an enterprise, meeting customer needs and achieving certain business results - i.e. strategy allows the organization to purposefully take advantage of its strengths and opportunities provided by the external environment and turn them into the future. The strategy is usually made with a time horizon of 3-5 years but can vary from 1 to 10 years.

Before a task can be included in the strategy, it is necessary to make a study of how modern technologies can support digital activities for the acquisition of new resources, digitalization, search, archiving, service, and other services. Based on this study, depending on the importance of each activity, it is possible to determine where to focus resources for the development of digitalization. In practice, there are often situations in which a new digitalization tool is first offered, the application of which is useful for the company, and then a plan for its acquisition is developed on its basis. Very often, experts from companies that offer such "technological solutions" argue that because the 
dynamics of the digital market is very high, it makes no sense to develop stand-alone strategies. At this stage, it is difficult to assess whether this claim is justified in relation to companies.

When developing the digitalization strategy, the Total Cost of Ownership method should be used to determine the financial costs - total cost of ownership throughout the period of use, as the costs of maintaining digitalization may be relatively high compared to the initial expenses for their acquisition. Finding the right balance between initial investment, maintenance costs and costs of working on strategic long-term projects is largely a matter of subjective skill on the part of the company's management, its intuition, knowledge, and experience.

The digitalization strategy, like a company's business strategy, must describe a desired future state, with deadlines and a plan for achieving the goals. The use of the latest hardware and software does not necessarily provide benefits if it does not comply with the digitalization strategy and does not support it [11]. Rather, these investments become an unnecessary expense and aggravate the company's budget not only in terms of acquisitions but also in terms of subsequent maintenance. Of course, the strategy needs to be developed and updated, as reasons of all kinds can arise that require change. It is possible that new tools will appear to increase the efficiency of services or to offer a new type of digital services. It is also possible that new means will emerge to reduce costs, such as the use of energyefficient computing technologies; occurrence of changes in the company's budget due to financial difficulties; opportunities to participate in or win a competition for a project under state funds; organizational change in the management of the company or in the organization to which it is organizationally subordinated, as usually each manager has his own views on the priorities and directions for development, etc.

The main difficulty in developing a strategy is the optimal use of existing infrastructure, software, and experience. If there is no significant compliance with them, then the implementation of the strategy would meet strong resistance from the company's employees, i.e. the people who will apply it. Another difficulty is the lack of qualified personnel on the labour market who are willing to work at the salaries typical for companies in our country. This poses major problems for the independent implementation of some projects. In our opinion, a promising direction in the development of software strategy is the transformation of digital services from a search tool to a tool for gaining knowledge about what and how users search, in order to use them to improve the search process of all users.

\section{Methods for Choosing Options for Digitalization}

The choice in each area of human activity of a specific solution among different options implies knowledge and search for a balance between different interrelated factors. Focusing just on one factor (criterion) or several factors can lead to wrong decisions, whether it is a technical [12] or business factor.

The choice of the specific solution from the available options is made based on criteria, requirements, and internal company standards. Each criterion has a different weight. There should be more than two options for each choice of options in order to have a multi-criteria problem that requires a decision. In a broad sense, the decision making is a process of identifying and choosing among options based on the preferences of the decision maker.

There are a lot of methods that have been developed to select the best of multiple options, each evaluated based on multiple criteria. Among the most popular methods [13] are:

- Technique for Order Preference by Similarity to the Ideal Solution (TOPSIS);

- The Analytical Hierarchy Process (AHP);

- ELimination Et Choice Translating REality (ELECTRE);

- Simple Additive Weighting (SAW);

- The Simple Multi Attribute Rating Technique (SMART);

- Preference Ranking Organization Method for Enrichment Evaluations (PROMETHEE), and so on.

In each of the considered techniques, we have many options, from which the best one should be chosen, and criteria based on which the choice is made. In order to arrange the options, three independent steps should be taken: 1) the criteria and the options are determined; 2) digital weights are assigned to the criteria; 3 ) the method for analysis of multiple criteria is applied, the calculation procedures are performed to determine the ranking of the options and the optimal solution is selected.

During the structuring of the problem, the goals and criteria are set, the alternatives, the constraints, the external environment and the stakeholders are defined; during the construction of the model the weights of the criteria are defined, quantitative and qualitative variables are derived; during the followup analysis, new options are created and sensitivity is analyzed.

The considered decision-making methods based on a combination of multiple criteria can be compared to outline the advantages and disadvantages of each and the area in which they are suitable for application.

For example, the main advantages of the Technique for Order Preference by Similarity to the Ideal Solution method, over the others [14] are: 
- Simple, rational and understandable concept;

- Ability to measure relative performance for each option in a simple mathematical form;

- Intuitive and clear logic, which represents the logic of human choice;

- Relatively easy calculation;

- The number of calculation steps remains the same regardless of the number of criteria.

The Technique for Order Preference by Similarity to the Ideal Solution method was developed by Huang and Chen [15] and is a multi-criteria method for choosing a solution from a finite set of alternatives. It is based on the concept that the chosen solution should have the shortest geometric distance from the positive ideal solution and the longest distance from the negative ideal solution. It is a compensatory method by which a bad result on one criterion is neutralized by a good result on another criterion. It calculates the results by comparing the Euclidean distances between the actual alternatives and the hypothetical alternatives. Finally, a numerical result is obtained to evaluate each of the selected alternatives. This is done in two stages: during the first stage decision makers identify and form a set of alternatives, criteria and variables that describe the problem to be solved. During the second stage, the method is applied to summarize the information from the first phase in a final evaluation.

To implement this method in an example scenario, we could assume that an organization is faced with a choice between several options for virtualization of the digital infrastructure for end users - through desktop virtualization and Solution A and Solution B have been developed by leading companies in virtualization.

When choosing a virtualization solution for the digital infrastructure of a specific organization, the mandatory criterion is the total cost of ownership of each of the alternatives. The choice of the number of years for which the costs are calculated is made by the decision makers [16], [17]. Other criteria can be infrastructure requirements (hardware and the information technology staff), availability, scalability of the solution, ease and reduction of administration time, and many others, specific to the particular organization.

The criteria selected by the decision maker in the organization for the purposes of the study are:

- Total cost of ownership - the total acquisition cost of the respective digital infrastructure option and the best criterion is the minimum total acquisition cost;

- Ease and reduction of administration time - for administration of desktop clients and the entire infrastructure, for updating desktop operating systems, for installing new software;

- Scalability - the ability to easily add new users without affecting the productivity of the existing ones;
- Availability - minimizing planned and unplanned stays so that the infrastructure is accessible to consumers when they need it;

- Infrastructure requirements (hardware and information technology staff) - the requirements are different for different solutions - higher for virtualized infrastructure and lower for traditional digital infrastructure.

Comparing alternatives and evaluating them through the weight of many criteria is a task that decision-makers - managers, owners, nongovernmental sectors, government and government services often face, and the future of organizations depends on the correctness of the choice. In the field of digitalization, the choice of solutions related to the digital infrastructure of companies determines the work of organizations at least a few years ahead.

Within our "Social media and digital interaction intelligence" project (the SoMeDi project) was advanced a natural language processing solution which allows advanced text analytics from various data sources [18].

Thus, the sentiment analysis microservice, implemented as a node.js application which can be consumed as a JSON REST API, was implemented in several use cases such as recruiting, online marketing campaigns analytics, and telecom market (analyzing customers' feedback). For the Human Resource Management use case, the goal was to improve the recruitment processes aiming to increase the efficiency of internship campaigns by ensuring a better match between the candidates' professional skills and the hiring company fields of activity [19].

We relate to this application as a real life scenario of digitalization strategy by referring the extensive work of analyzing the performance of the sentiment analysis engines (for example Google Cloud NLP, Azure Text Analytics, StanfordNLP and etc.) on various type of data, meaning, that the sentiment analysis engines, when tested and compared, have different performance depending on the text source domain - human resource management, marketing, banking, etc. So, we conclude that by applying a systematic design approach, using specific methods for choosing options for digitalization, we can enhance the outcomes of the digitalization process.

\section{Conclusion}

An integral part of the digital security system is the system for ensuring the continuity of digitalization services, which provides for the solution of various issues related to the restoration and ensuring the continuous supply of digitalization services. The use of multi-criteria decision-making methods puts the task in a mathematical framework and reduces the efforts of decision-makers. Different methods are suitable for solving different tasks and the study of their advantages and disadvantages allows them to choose the right method for the specific problem. 


\section{Acknowledgements}

This research was partially supported by NPI-45/2020 from the University of Economics - Varna Science Fund.

\section{References}

[1]. Kuyumdzhiev, I. (2019). Comparing Backup and Restore Eficiency in MySQL, MS SQL Server and MongoDB. International Multidisciplinary Scientific GeoConference: SGEM, 19(2.1), 167-174. doi: 10.5593/sgem2019/2.1/S07.022

[2]. Radev, M. (2019). Organizational Variants of IT Department at the University. In Conferences of the department Informatics (No. 1, pp. 284-290). Publishing house Science and Economics Varna.

[3]. Radev, M., \& Alexandrova, Y. (2013). Combining Virtualization Technologies in SOA-applications. In Proceedings of International Conference on Application of Information and Communication Technology and Statistics in Economy and Education (ICAICTSEE) (p. 56). International Conference on Application of Information and Communication Technology and Statistics and Economy and Education (ICAICTSEE).

[4]. Aleksandrova, Y. (2018). Application of Machine Learning for Churn Prediction Based on Transactional Data (RFM Analysis). In 18 International Multidisciplinary Scientific Geoconference SGEM 2018: Conference Proceedings (Vol. 18, No. 2.1, pp. 125-132).

doi: $10.5593 /$ sgem 2018/2.1/S07.016

[5]. Stoev, S. (2019). Using of additional packages of components for accelerated application development. Известия на Съюза на ученитеВарна. Серия Икономически науки, 8(2), 171-179. doi: 10.36997/IJUSV-ESS/2019.8.2.171

[6]. Petrov, P., Dimitrov, P., Stoev, C. A. P. D. S., Dimitrov, G. P., \& Bulut, F. (2020). Using the Universal Two Factor Authentication Method in Web Applications by Software Emulated Device. In 20 International Multidisciplinary Scientific Geoconference SGEM 2020: Conference Proceedings (Vol. 20, No. 2.1, pp. 403-410).

[7]. Pashev, G., Rusenova, L., Totkov, G., \& Gaftandzhieva, S. (2019). Business Process Modelling \& Execution Application in Work Education Domain. TEM Journal, 8(3), 992. doi: 10.18421/TEM83-42

[8]. Dimitrov, G. P., Panayotova, G., Garvanov, I., Os, B., Petrov, P., \& Angelov, A. (2016, September). Performance analysis of the method for social search of information in university information systems. In 2016 Third International Conference on Artificial Intelligence and Pattern Recognition (AIPR) (pp. 15). IEEE. doi: 10.1109/ICAIPR.2016.7585228

[9]. Panayotova, G., Dimitrov, G. P., Petrov, P., \& Os, B. (2016, September). Modeling and data processing of information systems. In 2016 Third International Conference on Artificial Intelligence and Pattern Recognition (AIPR) (pp. 1-5). IEEE. doi: 10.1109/ICAIPR.2016.7585229
[10].Vasilev, J., \& Stoyanova, M. (2019). Information Sharing With Upstream Partners of Supply Chains. International Multidisciplinary Scientific GeoConference: SGEM, 19(2.1), 329-336. doi: 10.5593/sgem2019/2.1/S07.043

[11].Petrov, P., \& Valov, N. (2019). Digitalization of banking services and methodology for building and functioning of Fintech companies. Izvestia Journal of the Union of Scientists-Varna. Economic Sciences Series, 8(1), 110-117. doi: 10.36997/IJUSV-ESS/2019.8.1.110

[12].Balabanova, I., Georgiev, G., Penchev, P., Kostadinova, S., \& Dimova, R. (2016, June). Classification of teletraffic service devices by $\kappa-\mathrm{NN}$, ANFIS and ANN classificators. In 2016 IEEE International Black Sea Conference on Communications and Networking (BlackSeaCom) (pp. 1-5). IEEE. doi:10.1109/BlackSeaCom.2016.7901585

[13].Velasquez, M., \& Hester, P. T. (2013). An analysis of multi-criteria decision making methods. International Journal of Operations Research, 10(2), 56-66.

[14].Hung, C. C., \& Chen, L. H. (2009, March). A fuzzy TOPSIS decision making model with entropy weight under intuitionistic fuzzy environment. In Proceedings of the international multiconference of engineers and computer scientists (Vol. 1, pp. 1316). IMECS Hong Kong.

[15].Cheng, S. J., \& Hwang, C. L. (1992). Fuzzy multiple attribute decision making: Methods and applications. Lecture Notes in Economics and Mathematical Systems, Springer.

[16].Petrova, S., Stefanov, S., Ivanov, S., Sergeev, A., \& Getova, I. (2019). Information systems used in Bulgarian university libraries as online public access catalogs. International Multidisciplinary Scientific GeoConference: SGEM, 19(2.1), 353-360. doi: $10.5593 /$ sgem2019/2.1/S07.046

[17].Petrova, S., Sergeev, A., Getova, I., \& Kostadinova, I. S. (2019). Online Public Access Catalogs in Bulgarian University Libraries an Empirical Study of Seven-Year Evolution. In 12th Annual International Conference of Education, Research and Innovation (ICERI) (pp. 230-236). International Academy of Technology, Education and Development. doi: 10.21125/iceri.2019.0091

[18]. Vasilescu, C., Suciu, G., \& Pasat, A. (2019). A New Method to Help the Human Resources Staff to Find the Right Candidates, Based on Deep Learning. In The International Scientific Conference eLearning and Software for Education (Vol. 3, pp. 240-246). " Carol I" National Defence University. doi: 10.12753/2066-026X-19-170

[19].Suciu, G., Boscher, C., Prioux, L., Pasat, A., \& Dobre, C. (2017). Insights into Collaborative Platforms for Social Media Use Cases. Studies in Informatics and Control, 26(4), 435-440. doi: $10.24846 / \mathrm{v} 26 \mathrm{i} 4 \mathrm{y} 201707$ 